\title{
El campo de los estudios del juego
}

The field of the game studies

O campo de estudos de jogos

\section{EDITORIAL}

\section{Ruth S. Contreras Espinosa}

Universitat de Vic - Universitat Central de Catalunya

Profesora del Departamento de Comunicación. Editora de la revista Obra Digital.

ruth.contreras@uvic.cat

orcid.org/0000-0002-9699-9087

\section{Resumen:}

Los estudios del juego han sido concebidos básicamente como un campo interdisciplinario que ha intentado desarrollar un marco amplio para el estudio de los juegos (aquí se incluyen videojuegos, juegos de mesa e historias interactivas). En ellos se han descrito experiencias de desarrolladores y de las comunidades que los rodean, así como ontologías, metodologías, etc. Sin embargo, es necesario continuar investigando en el área de los juegos, integrando nuevas direcciones de los estudios con la intención de abrir el campo a nuevas ideas y personas.

\section{PALABRAS CLAVE}

Videojuegos, Sociedad digital, Estudios del juego.

\begin{abstract}
:
Game studies have basically been conceived as an interdisciplinary field that has attempted to develop a broad framework for the study of games (this includes video games, board games and interactive stories). They have described the experiences of developers and the communities that surround them, as well as ontologies, methodologies, etc. However, it is necessary to continue researching around games, integrating new directions of studies with the intention of opening the field to new ideas and people.
\end{abstract}

\section{KEYWORDS}

Video games, Digital society, Game studies. 


\section{Resumo}

Os estudos de jogos foram concebidos basicamente como um campo interdisciplinar que tentou desenvolver uma ampla estrutura para o estudo de jogos (incluindo videogames, jogos de tabuleiro e histórias interativas). Eles descreveram experiências de desenvolvedores, das comunidades que os cercam, bem como ontologias, metodologias etc. No entanto, é necessário continuar pesquisando na área de jogos, integrando novos rumos de estudos com a intenção de abrir o campo para novas ideias e pessoas.

\section{PALAVRAS-CHAVE}

Videogame, sociedade digital, estudo de jogos.
Los estudios del juego o game studies pueden verse como una corriente de investigación sobre videojuegos, juegos digitales y juegos en general que llega a principios de la década de 2000 con el auge de los videojuegos como pasatiempo popular, pero además se une a este auge el hecho de que se hayan consolidado algunas revistas académicas (Stenros \& Kultima, 2018). Concebida como un área interdisciplinaria y con investigadores expertos en muy diversos campos de estudio, ha intentado definir un marco coherente para estudiar los juegos en donde diseñadores, desarrolladores y académicos describen sus experiencias, metodologías, previsiones y análisis, entre otras cosas, y dan visibilidad e importancia a las comunidades que surgen alrededor de los juegos.

En los últimos años se han realizado algunos intentos para medir el alcance de los temas investigados en este campo. Por ejemplo, se han realizado análisis bibliométricos con la intención de establecer datos y detectar cuáles son los temas más investigados o las comunidades que se observan alrededor de ellos (Melcer et al., 2015), además de clasificar los juegos y sus géneros derivados (Coavoux et al., 2017). Pero, aunque con el tiempo hayan crecido estas voces que incluyen la investigación de diversos juegos (no solo digitales) sino también todos aquellos fenómenos adyacentes, es evidente que aún contamos con una investigación limitada en el estudio de juegos. Faltan estudios con énfasis en estudios cognitivos, inteligencia artificial, aprendizaje informal (Scolari \& Contreras-Espinosa, 2019) o en otras áreas mas técnicas.

Deterding (2017) destaca que los game studies se formaron inicialmente para ser una disciplina "paraguas", que se ha convertido en una subcomunidad dentro de la investigación de juegos. Además, señala que el éxodo de académicos de las áreas de la comunicación y la psicología a esta área ha servido para ampliar el estudio de los juegos, con lo que hemos ganado en nuevas investigaciones, enfoques y puntos de vista. Básicamente, los estudios del juego hoy en día cuentan con académicos de áreas como las humanidades, las ciencias sociales, la comunicación, el diseño centrado en el usuario, etc. y un gran grupo de adeptos a su estudio como fenómeno cultural. Deterding sugiere además que en este campo ha ocurrido algo similar a lo que ocurrió con el cine, al abordar predominantemente lo social y la cultura, y después ir a cuestiones mucho más técnicas.

Pero debemos tomar en cuenta que hace falta también revisar el panorama de los juegos actuales, así como la variedad de fenómenos que ahora se incluyen en el concepto "juego". Podrían incorporarse en la definición no solo los videojuegos, los juegos de mesa o las historias interactivas. Falta incorporar los esports y muchas otras actividades que no se clasifican fácilmente como juegos pero que actualmente 
comparten algunos aspectos o infraestructura digital. Los conceptos y clasificaciones creados hace años alrededor del juego puede que sean limitantes o poco amplios para las dinámicas establecidas actualmente y para las prácticas de juego que utilizan los jugadores ahora. Además, falta ahondar en la situación de la mujer en los juegos, en otros colectivos (Fron et al., 2007), en las razas (Gray, 2012) y en las situaciones laborales de los trabajadores de la industria (Hammar et al. 2020). Es necesario alejarnos de clasificaciones binarias y visualizar un camino hacia las clasificaciones fluidas e inclusivas.

Incluso es necesario investigar nuevas metodologías, tanto en el sentido de enfoques sobre cómo analizar los juegos, o en cómo debemos seleccionar juegos que queremos analizar. Coavoux et al. (2017) indican que en cualquier campo de investigación, especialmente en la cultura o en las artes, los gustos personales de los investigadores son muy importantes en la elección de los objetos de investigación. Por lo tanto, al profundizar en las metodologías utilizadas actualmente en las investigaciones relacionadas con juegos, estaríamos teniendo en cuenta esa ruta que siguen los investigadores. Considero que debemos abrirnos a nuevos conceptos, aceptar nuevas elecciones de los investigadores, pero además incluir nuevas metodologías para concebir nuevos estudios. De acuerdo con Pettman (2019), deberíamos adoptar un nuevo enfoque para examinar los procesos y condiciones que nos permitan estudiar los juegos de otra forma. Podríamos ver el otro lado de la moneda integrando nuevas direcciones de los estudios, de la misma manera que se ha hecho con otras áreas con mayor tradición. Esto ayudaría incluso a reducir la barrera percibida para los recién llegados a estos estudios, pues abriría el campo a nuevas ideas y personas.
Los artículos contenidos en este monográfico representan un testimonio de esa necesidad de continuar investigando en el área de los juegos. Por ello nos dimos a la tarea de hacer un hueco al área de los game studies y a nuevos rostros en la revista Obra Digital. El lector encontrará una gran variedad de artículos que estudian el videojuego desde el punto de vista de la educación, un análisis sobre la estereotipación de la bisexualidad, la gestión de una comunidad política, o la importancia del medio lúdico para favorecer ideologías. Se incluye asimismo en el monográfico un estudio sobre el empoderamiento de las mujeres mediante el análisis la obra de The Last of US parte /l y se reflexiona sobre cómo las narrativas promueven un pensamiento crítico. Finalmente, se destaca la importancia de Flash en la configuración de diversos movimientos artísticos ligados al ámbito virtual.

Se trata de un numero muy especial para nosotras no solo por los artículos reunidos en el monográfico, sino además porque a partir de este número la revista Obra digital cambiará su equipo y actividad. De la misma manera que los estudios del juego se enfrentaron a cambios, esta ha de definir un marco nuevo de trabajo. Los cambios son inevitables pero necesarios para dar sentido a nuestros objetivos. Solo me queda por decir: muchas gracias a todos los lectores y autores que nos han acompañado en todos estos años. No quiero cerrar sin dar las gracias públicamente a la Dra. Cecilia Ugalde Sánchez, mi amiga y colega; gracias por todo su trabajo y constante implicación a lo largo de esta etapa. Espero que disfruten este número dedicado a los videojuegos tanto como nosotras hemos disfrutado editándolo. 


\section{REFERENCIAS}

Coavoux, S., Boutet, M., \& Zabban, V. (2017). What We Know About Games: A Scientometric Approach to Game Studies in the 2000s. Games and Culture, 12(6), 563-584. https://doi.org/10.1177/1555412016676661

Deterding, S. (2017). The Pyrrhic Victory of Game Studies: Assessing the past, Present, and Future of Interdisciplinary Game Research. Games and Culture, 12(6), 521-543. https://doi.org/10.1177/1555412016665067

Fron, J., Fullerton, T., Morie, J. F., \& Pearce, C. (2007). Playing Dress-Up: Costumes, roleplay and imagination. Philosophy of Computer Games Conference, Universidad de Gales (Reino Unido). http://gamephilosophy. org/wp-content/uploads/confmanuscripts/pcg2007/Pearce_PaperPCG2007.pdf

Gray, K. L. (2012). Deviant bodies, stigmatized identities, and racist acts: Examining the experiences of African-American gamers in Xbox Live. New Review of Hypermedia and Multimedia, 18(4), 261-276. https://doi.org/10 $.1080 / 13614568.2012 .746740$

Hammar, E. L., Wildt, L. de, Mukherjee, S., \& Pelletier, C. (2020). Politics of Production: Videogames 10 years after Games of Empire. Games and Culture. https://doi.org/10.1177/1555412020954996

Melcer, E. F., Nguyen, T.-H. D., Chen, Z., Canossa, A., El-Nasr, M. S., \& Isbister, K. (2015). Games research today: Analyzing the Academic Landscape. Proceedings of the 10th International Conference on the Foundations of Digital Games (FDG 2015), Pacific Grove (EE. UU.).

Pettman, D. (2021, noviembre 23). The Species Without Qualities: Critical Media Theory and the Posthumanities. B2O the online community of the boundary 2 editorial collective. https://www.boundary2.org/2019/04/ the-species-without-qualities-critical-media-theory-and-the-posthumanities/

Scolari C. A., \& Contreras-Espinosa, R. S. (2019). How do teens learn to play video games? Informal learning strategies and video game literacy.

Journal of Information Literacy. 13(1), 45-61. https://doi.org/10.11645/13.1.2358

Stenros, J., \& Kultima, A. (2018). On the Expanding Ludosphere. Simulation \& Gaming, 49(3), 338-355. https://doi.org/10.1177/1046878118779640 